\title{
Radio Aurora Explorer: A Mission Overview
}

\author{
James W. Cutler* \\ University of Michigan, Ann Arbor, Michigan 48109 \\ and \\ Hasan Bahcivanı̊ \\ Center for Geospace Studies, SRI International, Menlo Park, California 94025-3493
}

DOI: $\underline{10.2514 / 1 . A 32436}$

\begin{abstract}
The primary objective of the Radio Aurora Explorer mission is to study plasma instabilities that lead to magnetic field aligned irregularities of electron density in the lower polar ionosphere $(80-400 \mathrm{~km})$. These irregularities are known to disrupt communication and navigation signals. The Radio Aurora Explorer mission uses a bistatic radar configuration; the spacecraft is the radar receiver and the transmitters are several worldwide incoherent scatter radars of which the primary radar is located in Poker Flat, Alaska. The satellites are $3 \mathrm{~kg}$, university-class CubeSats with a primary payload of a direct-conversion radar receiver operating in the frequency range from 426 to $510 \mathrm{MHz}$. The satellite bus has a novel configuration of processing, attitude control, and antenna design that met the physical constraints of the CubeSat form factor and the one-year schedule constraint imposed by launch dates. Two satellites have launched: RAX-1 on 19 November 2010 onboard a Minotaur-IV rocket from Kodiak, Alaska, and RAX-2 on 28 October 2011 onboard a Delta-II rocket from Vandenberg U.S. Air Force Base, California. RAX-2 has detected and measured irregularities during multiple experiments over Alaska and Canada. Preliminary analysis indicates unprecedented characterization of the irregularities.
\end{abstract}

\section{Introduction}

$\mathbf{T}$ HE Radio Aurora Explorer (RAX) mission is the first nanosatellite mission commissioned by the National Science Foundation to study space weather [1]. In 2007, a workshop was held to explore the potential use of a specific nanosatellite form factor, the CubeSat [2], for novel space weather missions. Despite their obvious limitation in mass and size, these small satellites offer potential advantages that compliment their larger forerunners [3]. They provide the opportunity for rapid implementation and deployment of sensors in space; for focused and narrow science missions that target a specific space weather phenomenon; and for increased multipoint measurements of space processes through coordinated and distributed satellites. Their low cost encourages creative, higher-risk missions and their small form factor and weight provide faster launch opportunities than larger spacecraft [4].

The RAX mission is studying meter-scale ionospheric electron density fluctuations, called field-aligned irregularities (FAI). These irregularities are capable of scattering radio signals, which can be deleterious for space-based communication and navigation [5]. For example, space-based surveillance radars can encounter this form of scatter as "radar clutter," which could mask the detection of targets of interest. In most cases, direct mitigation of plasma-induced disturbances is not a viable or even feasible approach. Hence, understanding of the processes that lead to the generation and distribution of plasma structure will enable short-term forecasting that predicts when disruptions will occur and give satellite operators the ability to alter communications according to these forecasts.

The RAX mission is a joint effort between SRI International and the Michigan Exploration Laboratory (MXL) at the University of Michigan. Instrument development occurred at SRI and spacecraft design, building, and integration occurred at MXL. Work on RAX began in late 2008 and currently, two satellites are in orbit: RAX-1, launched in November 2010, and RAX-2, launched in October 2011.

Received 4 June 2012; revision received 9 January 2013; accepted for publication 11 January 2013; published online 25 June 2013. Copyright (C) 2013 by James W. Cutler and Hasan Bahcivan. Published by the American Institute of Aeronautics and Astronautics, Inc., with permission. Copies of this paper may be made for personal or internal use, on condition that the copier pay the $\$ 10.00$ per-copy fee to the Copyright Clearance Center, Inc., 222 Rosewood Drive, Danvers, MA 01923; include the code 1533-6794/13 and $\$ 10.00$ in correspondence with the CCC.

*Assistant Professor, Department of Aerospace Engineering.

${ }^{\dagger}$ Research Physicist.
Science operations are managed at SRI and satellite operations take place at MXL. Ground stations around the world partner to downlink both science and telemetry data from the RAX satellites.

Previously, Bahcivan and Cutler discussed the RAX mission with an emphasis on the science mission and radar system [6]. The contributions of this paper include a focused analysis of the satellites and a holistic discussion of the entire mission system. First, a summary is given of the innovative bistatic radar configuration that provides measurements to gain a fundamentally new insight into space weather processes related to auroral FAI. Second, a mission architecture is identified that harnesses the potential to perform cutting-edge science while adhering to the constraints of nanosatellites. Third, a description of design approaches and philosophies that are able to accommodate an extremely aggressive delivery schedule and limited funding, all while in an educational environment, is provided. Initial flight status is given, and the paper concludes with a summary of contributions.

\section{Scientific Background and Objectives}

The primary scientific objective of the RAX mission is to study lower ionospheric processes, specifically the microphysics of plasma instabilities that lead to FAI of electron density in the polar lower (80$400 \mathrm{~km}$ ) ionosphere through high-resolution mapping of auroral ionospheric irregularities for a wide range of ionospheric currents. High-latitude polar measurements of the irregularities have not been possible due to geometric limitations in remote sensing capabilities and insensitive in-situ measurements. Unlike the FAI in the equatorial ionosphere, high horizontal and altitudinal resolution measurements of high-latitude irregularities have not been possible. This is primarily caused by the difficulty in achieving a scattering geometry normal to magnetic field lines, which are nearly vertical at high latitudes. Coherent scatter radars can be pointed at very low elevations to scatter off irregularities at normal angles, however, these radars cannot make height-resolved measurements because either the beam width in the vertical direction is too wide or refraction causes source location ambiguity. In-situ measurements are also difficult because ionospheric altitudes below $300 \mathrm{~km}$ are beyond the reach of orbiting spacecraft. Sounding rockets have measured irregularities with the highest altitude resolution, yet they are not sufficiently sensitive to distinguish energy flow with respect to magnetic aspect angle. Thus, for further FAI studies, a unique approach is required for enhanced spatial resolution measurements. 
The RAX mission overcomes the limitations of existing measurements by using a novel ground-to-space bistatic radar experimental configuration. The ground-to-space bistatic geometry enables high horizontal and altitudinal resolution measurements of the auroral FAI. The high resolution is achieved through two means: 1) the radar illuminating the irregularities has a narrow beam width, which is typical of all incoherent scatter radars (ISR); and 2) the radar-to-irregularity distance is short because the radar can be pointed at high elevation angles, as opposed to a monostatic radar pointed at very low elevation in order to meet the perpendicularity condition.

The RAX mission will provide insight into the fundamental physics of FAI formation by enabling measurement of several plasma wave types and related turbulence. The specific conditions leading to irregularity formulation are not well understood, thus rendering the prediction of FAI characteristics not yet possible. However, it is known that large electric currents in the $\mathrm{E}$ and $\mathrm{F}$ regions of the magnetosphere-ionosphere circuit excite plasma instabilities that lead to meter-scale irregularities. Within these currents, observations made by radar, rocket, and satellite have shown the existence of waves in the form of plasma turbulence.

In the E region, the strongest waves are associated with the FarleyBuneman instability [7-9]. Many authors formulated the instability with a linear dispersion relation, such as Fejer et al. [10]. The linear dispersion relation partially explains wave features, such as the phase velocity and growth rate, for small amplitudes. For example, the instability is excited when the convection electric field $E_{c}$ exceeds a threshold of approximately $20 \mathrm{mV} / \mathrm{m}$. This often occurs at high latitudes. However, when the waves are strongly driven by a greater electric field, they grow too large in amplitude and saturate [11], a process that is not sufficiently understood to predict the wave features. The RAX mission will provide additional data for assessing this strongly driven wave phenomenon.

In the $\mathrm{F}$ region, the RAX mission has the potential to measure meter-scale waves, including electrostatic ion cyclotron (EIC) and Post-Rosenbluth waves. EIC waves are considered likely plasma wave modes in and near auroral arcs because they have the lowest threshold among the various current-driven instabilities that can be excited by field-aligned currents [12]. In-situ satellite measurements suggest that EIC waves can be destabilized and grow to large amplitudes [13]. Ott and Farley [14] predicted that high-latitude convection would often be strong enough to excite the PostRosenbluth instability $[15,16]$. The instability leads to submeter-scale $(10-20 \mathrm{~cm})$ field-aligned irregularities; therefore, an ultra-highfrequency (UHF) radar should detect the waves. The bistatic radar configuration of the RAX mission eliminates the geometry concerns for remote sensing and may be able to measure these previously undetected waves.

\section{Science Measurement Methods}

The RAX mission is specifically designed to remotely measure, with high angular resolution $(\sim 0.5 \mathrm{deg})$, the three-dimensional (3D) $k$-spectrum (spatial Fourier transform) of $\sim 1$-m-scale FAI. The spacecraft will measure the signal strength of radio aurora [17], the scattered signals from irregularities that are illuminated by narrow beam transmissions from a ground-based incoherent scatter radar. The scattering locations are determined using time and position synchronization between radar transmissions and satellite receptions with the assumption that the scattering occurs only inside the narrow radar beam. Time and position are referenced to data from the GPS. This measurement method uses a bistatic radar [18], where the radar is the transmitter and the satellite is the receiver. Figure 1 shows the measurement concept with the irregularities, magnetic field lines, radar beam, radio aurora (cones) and the satellite (cubes), as well as the satellite path. The ground radar illuminates ionospheric irregularities with high-power radar pulses. The irregularities inside the narrow radar beam at a given altitude scatter the signals in a hollow cone shape. The thickness of the wall of each cone is a measure of magnetic aspect sensitivity, which is also a measure of plasma wave energy distribution in the parallel and perpendicular directions with respect to the geomagnetic field. The RAX CubeSats fly through the echoed

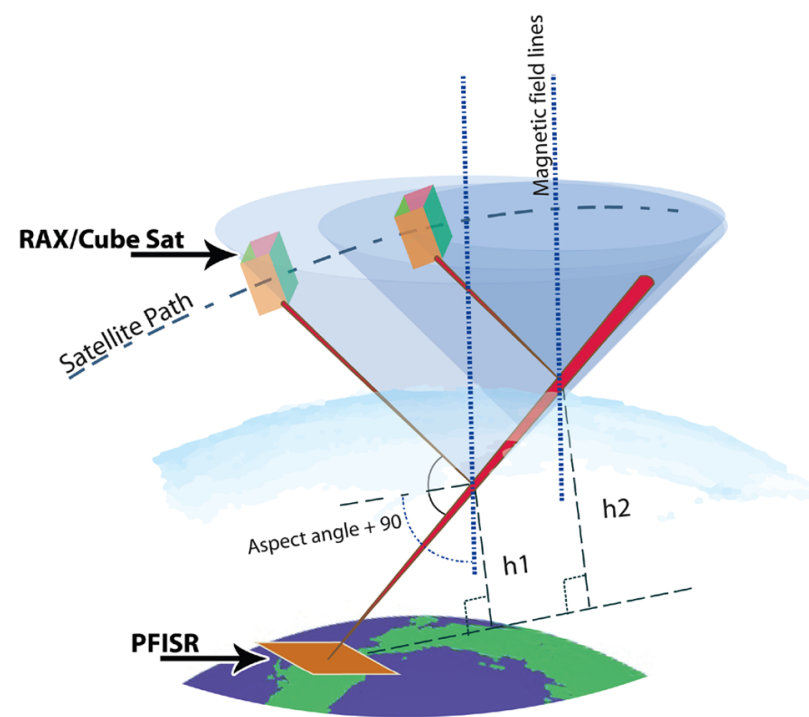

Fig. 1 Measurement concept of operation for the RAX mission.

signals and measure their intensity. The two cube figures represent a single satellite passing in time through the experimental zone.

The main RAX science product is $I\left(E_{c}, N_{e}, T_{e}, T_{i}, h, \theta\right)$; that is, plasma irregularity intensity $I$ as a function of convection electric field $E_{c}$, electron density $N_{e}$, electron and ion temperatures $T_{e}, T_{i}$, altitude $h$, and magnetic aspect angle $\theta$. RAX measures $I$, the ground radar measures the plasma parameters $\left(E_{c}, N_{e}, T_{e}, T_{i}\right)$, and $(h, \theta)$ are given by the experimental geometry. It should be noted that these measurements are dependent on the transmission and receiving times as well, which are determined from GPS time. The radar returns will be analyzed to identify the waves responsible for radar scatter and determine the ionospheric conditions leading to the corresponding plasma instabilities.

\section{A. Incoherent Scatter Radars}

The RAX radar receiver is compatible with the five UHF radars shown in Table 1 . The receiver can also receive transmissions from the Modular UHF Ionospheric Radar (MUIR) located at the HighFrequency Active Auroral Research Program (HAARP) facility in Gakona, Alaska, as part of active ionospheric heating experiments. However, the sensitivity to high-frequency (HF) heater-induced artificial FAI has not yet been calculated. In addition, radars with electronic beam steering capability, like the Poker Flat ISR (PFISR) and Resolute ISR (RISR) can be used for simultaneous multiple beam position experiments that enable FAI measurements at multiple locations during a single pass.

\section{B. Payload Receiver}

The RAX payload receiver design leveraged extensive heritage from the Advanced Modular Incoherent Scatter Radar design [19]. The receiver is a direct-conversion (homodyne) design using industrial-grade components. To receive the low-signal strength echo from the irregularities, the receiver is extremely sensitive, with a noise floor of $-114 \mathrm{dBm}$. The RAX receiver also exhibits extremely

Table 1 Incoherent scatter radars operating at UHF that are RAX compatible

\begin{tabular}{lcccc}
\hline \hline Radar & $\begin{array}{c}\text { Frequency, } \\
\text { MHz }\end{array}$ & $\begin{array}{c}\text { Power, } \\
\text { MW }\end{array}$ & $\begin{array}{r}\text { Beam width, } \\
\text { deg }\end{array}$ & $\begin{array}{c}\text { Invariant latitude, } \\
\text { deg }\end{array}$ \\
\hline PFISR & 449 & 2.0 & 1.0 & 78 \\
RISR & 443 & 2.0 & 1.0 & 81 \\
Svalbard & 500 & 1.0 & 0.6 & 75 \\
Millstone & 440 & 2.5 & 0.6 & 53 \\
Arecibo & 430 & 2.5 & 0.2 & 34 \\
\hline \hline
\end{tabular}




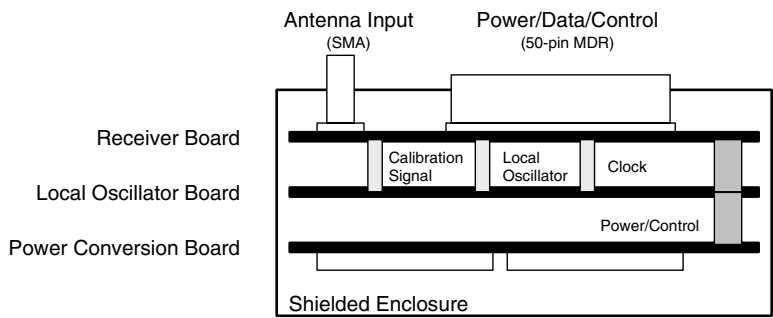

Fig. 2 A block diagram of the RAX radar receiver.

wide dynamic input range of over $60 \mathrm{~dB}$, rapid overload recovery in less than $10 \mu \mathrm{s}$, power consumption of less than $2.6 \mathrm{~W}$, efficient internal heat dissipation, stable long-term frequency lock, and robust shielding from electromagnetic interference. The input noise temperature is approximately $400 \mathrm{~K}(3.8 \mathrm{~dB}$ noise figure). The receiver consists of three circuit boards contained in an aluminum housing that provides RF shielding and thermal pathways. A block diagram is shown in Figure 2.

In-phase and quadrature components $(I$ and $Q)$ of the received baseband signal are digitized to 14-bit resolution, which enables sophisticated digital signal processing onboard the satellite. The receiver outputs this digital data at $1 \mathrm{MHz}$ with $I$ and $Q$ interleaved on rising and falling edges of the output clock. An additional output bit specifies if the analog-to-digital conversion has overflowed from direct radar beam reception. A $500 \mathrm{MHz}$ onboard signal generator provides a $-30 \mathrm{dBm}$ calibration signal for on-orbit characterization.

The radar receiver was designed to receive pulse transmissions from the radars in Table 1. It contains preselection filters and a tunable local oscillator to allow operation from 426 to $510 \mathrm{MHz}$ in the following megahertz bands: 426-434, 437-445, 443-452, and 483510. Expected received signal strengths are compatible with the receiver sensitivity [20].

\section{Payload Operations}

During a radar measurement experiment, the payload receiver operates in a snapshot acquisition mode, collecting raw samples at $1 \mathrm{MHz}$ for $300 \mathrm{~s}$ over the experimental zone. This generates $32 \mathrm{Mbps}$, for a total of $1.2 \mathrm{~GB}$ of data per snapshot experiment. Following each experiment, the raw data samples are postprocessed onboard the satellite for range-time intensity and Doppler spectrum.

The mission is capable of two to three experiments per day with primary limitations on experiments due to data downlink rates. Figure $\underline{3}$ shows simulated one-minute tracks that pass through an experimental scattering zone. For a given altitude and magnetic aspect angle, the satellite will cross a cone at two points during a single experiment. For an expected year lifetime, there are approximately 1000 passes that satisfy geometry constraints for the bistatic experiments. However, based on electric field statistics from the DE2 satellite [21], it is estimated that only one out of five passes will have sufficient field strength for irregularity formation. Thus, experimental runs will occur as often as possible to provide sufficient data collections for statistically significant results.
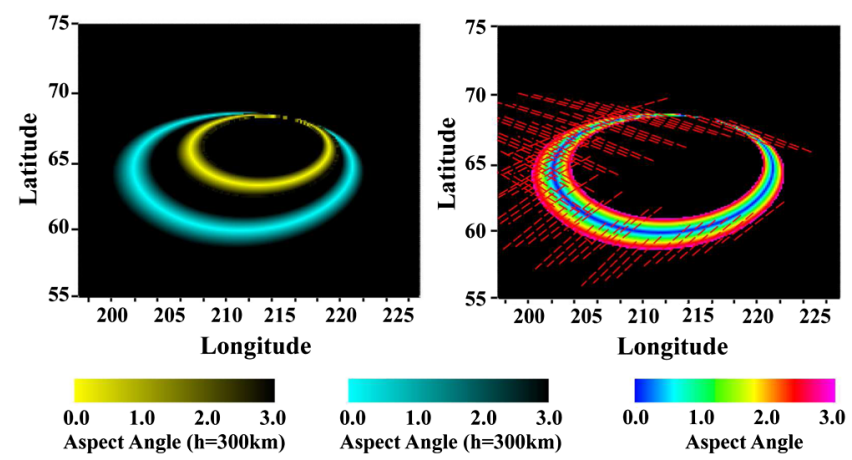

Fig. 3 Example scattering zones at different altitudes are shown on the left overhead of PFISR.

\section{Satellite Design}

In this section, the RAX satellites, classified as university-class satellites [22], are described. First, a summary of primary mission requirements is given, which are derived from the science objectives and which guide the design and implementation of the satellites. Next, the high-level satellite architecture and its achievements of specific requirements are described. A brief overview of satellite subsystems is given as well. Full subsystem details are left for future work.

\section{A. Mission Requirements}

Four top-level mission requirements are derived from the science mission objectives described in Sec. III. The first requirement is that 300 experiments shall be run during the first year of RAX operation. This quantity of experiments is expected to provide statistically significant results relative to the expected occurrence of irregularities. The second requirement is that received radar data shall be timetagged to GPS time within one microsecond. This ensures proper phase decoding of received radar pulses and improves range resolution (distance determination) to the scattering irregularities. The third requirement is that the antenna gain relative to the target scattering regions shall be known within $1 \mathrm{~dB}$. RAX's primary measurement is intensity $I$ of irregularity radar echoes. The gain of the receive antenna is directly proportional to the received signal strength. This $1 \mathrm{~dB}$ requirement limits the uncertainty in measurement of the radar echoes and improves science analysis. The fourth requirement is that RAX shall be designed such that simulated deorbit times are within 25 years after end of mission. The U. S. Federal Communications Commission limits the orbital lifetime of satellites by requiring space objects to deorbit 25 years after end of mission. Within all the aforementioned requirements, the satellite also had to conform to the unyielding restrictions of the CubeSat form factor (dimensions of $30 \times 10 \times 10 \mathrm{~cm}$ ) and a time-to-launch of less than a year; RAX-1 was manifested for launch with a delivery date 11 months after program start.

\section{B. Satellite Overview}

To satisfy these requirements and the initial mission constraints, the RAX satellites were developed as $3 \mathrm{U}$ CubeSats, confined to the dimensions of $30 \times 10 \times 10 \mathrm{~cm}$, and pictured in Fig. 4 . Four of the satellite faces $(+X,+Y,-X,-Y)$ contain body-mounted solar panels, each with an integrated attitude determination sensor suite. RAX-1 panels each contain a dual axis magnetometer, a coarse sun sensor, and a temperature sensor. RAX-2 panels contain three coarse sun sensors and a temperature sensor. On both satellites, the $+X$ panel also contains three external connections for reprogramming internal computers after integration. The top face $(+Z)$ has four UHF antennas, an S-band patch antenna, and three coarse sun sensors. The bottom face $(-Z)$ has a GPS patch antenna and two coarse sun sensors.
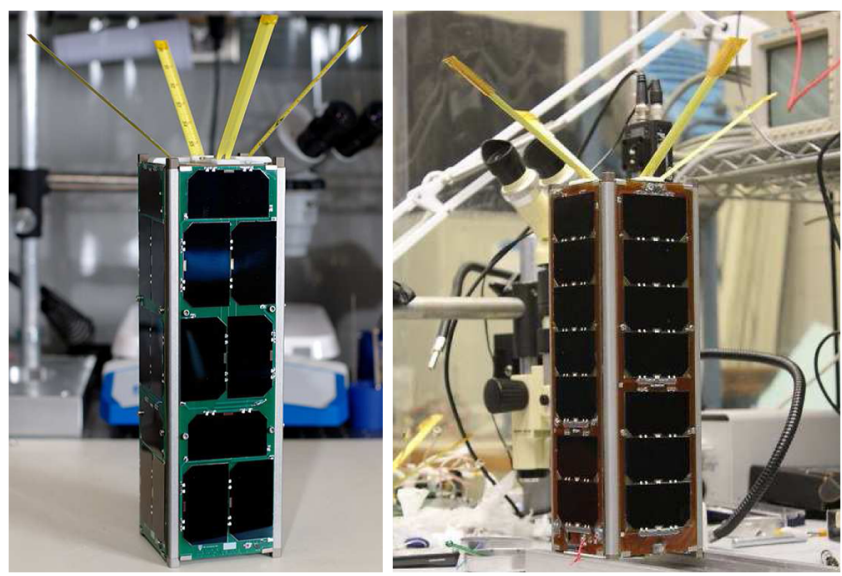

Fig. 4 The RAX satellites: RAX-1 is on the left; RAX-2 is on the right. 


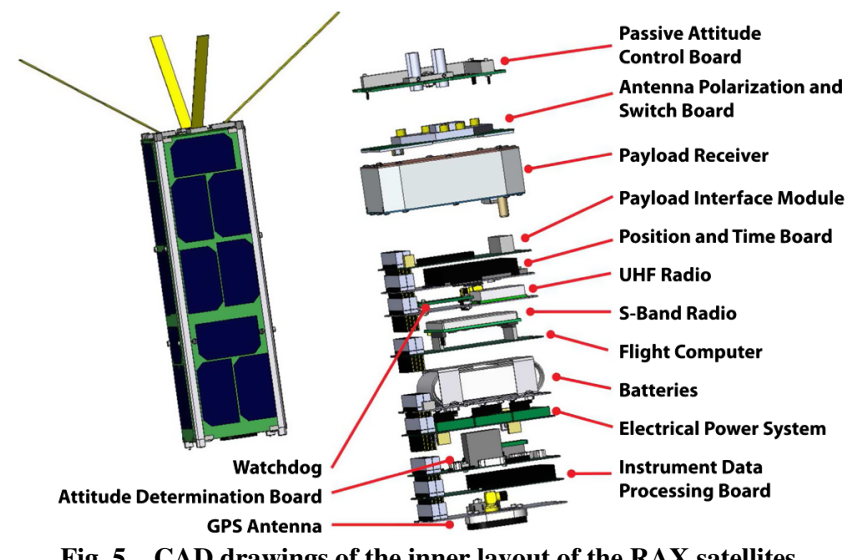

Fig. 5 CAD drawings of the inner layout of the RAX satellites.

The internal layout of the satellite subsystems is shown in Fig. 5. The boards are integrated vertically with the board plane parallel to the $Z$ axis and connected with a 104-pin connector. The stacking above the payload interface module has a custom configuration due to the payload receiver and complexity of the upper subsystems. The masses of RAX-1 and RAX-2 are 2.8 and $2.9 \mathrm{~kg}$, respectively. A summary of the satellite components is given in Table $\underline{2}$ with details in the next sections.

RAX-2 contained several upgrades to RAX-1 based on RAX-1 orbit performance. Solar panels were redesigned to fix a design flaw that resulted in a premature mission termination for RAX-1. Additional sun sensors were added to improve attitude determination. Minor structural modifications were made to improve battery mount performance during vibration testing. Summaries of the changes are given in later sections.

\section{Major Design Considerations}

Beyond normal satellite design efforts for systems such as power and communication, there were two major payload-specific design activities. First, a properly pointed antenna system was needed to satisfy the third mission requirement: $1 \mathrm{~dB}$ knowledge of the antenna gain pattern with respect to the scattering regions. Second, sufficient storage and processing were needed for data from the payload receiver: $1.2 \mathrm{~GB}$ of data output at a rate of $32 \mathrm{Mbps}$ for each fiveminute experiment.

\section{UHF Antenna Design and Attitude Control}

The design process began with the antenna gain requirement. The bistatic radar geometry and environmental parameters were analyzed over the primary radar transmitter, PFISR. In the experimental region above PFISR, Earth's magnetic field is nearly vertical with a deviation from local vertical less than $20 \mathrm{deg}$.

Combining this environmental magnetic feature with two wellknown satellite technologies, passive magnetic stabilization and a turnstile antenna, a purely passive attitude control system and antenna was developed that theoretically satisfies the $1 \mathrm{~dB}$ antenna gain requirement of the science instrument while meeting the constraints of the CubeSat form factor. This system was augmented with an attitude determination system to account for any implementation-related uncertainties that might introduce error and nonuniformities into the antenna system.

With a relatively simple passive magnetic control system, the RAX satellites are designed to maintain a near nadir-pointing attitude over the PFISR experimental zone. A permanent magnet aligns the satellite with Earth's magnetic field. Magnetic hysteresis material dampens a majority of rotational kinetic energy that the satellite obtained from initial launch vehicle deployment. This system provides two-axis control; the satellite is free to rotate around the $Z$ axis, spinning along the magnetic field line. The control-system simulations predict a maximum control error from the local magnetic field of \pm 4 deg with a \pm 3 deg oscillation around that error, giving a total of $\pm 7 \mathrm{deg}$ from the local magnetic field. Simulations of this control system implemented in representative orbits allow the team to bound the expected arrival angles of radar signals to RAX. Figure $\underline{6}$ plots expected arrival angles of radar echoes given the bistatic radar geometry and the magnetic field alignment. Angles are from five regions, north, south, east, and west of PFISR, as well as one centered directly above.

With this analysis, the requirements for the payload antenna are derived. First, it must be symmetric around the $Z$ axis of the satellite, eliminating the limitations of two-axis control. Second, it must have less than $1 \mathrm{~dB}$ of antenna gain throughout the expected signal arrival angles and an uncertainly in pointing of $\pm 77 \mathrm{deg}$. A turnstile antenna system was developed that meets these requirements [23]. Thus, with a purely passive system consisting of passive magnetic control and a turnstile antenna, the RAX satellites provide antenna gain knowledge within $1 \mathrm{~dB}$ for arriving radar pulses.

Table 2 Summary of RAX satellite subsystems

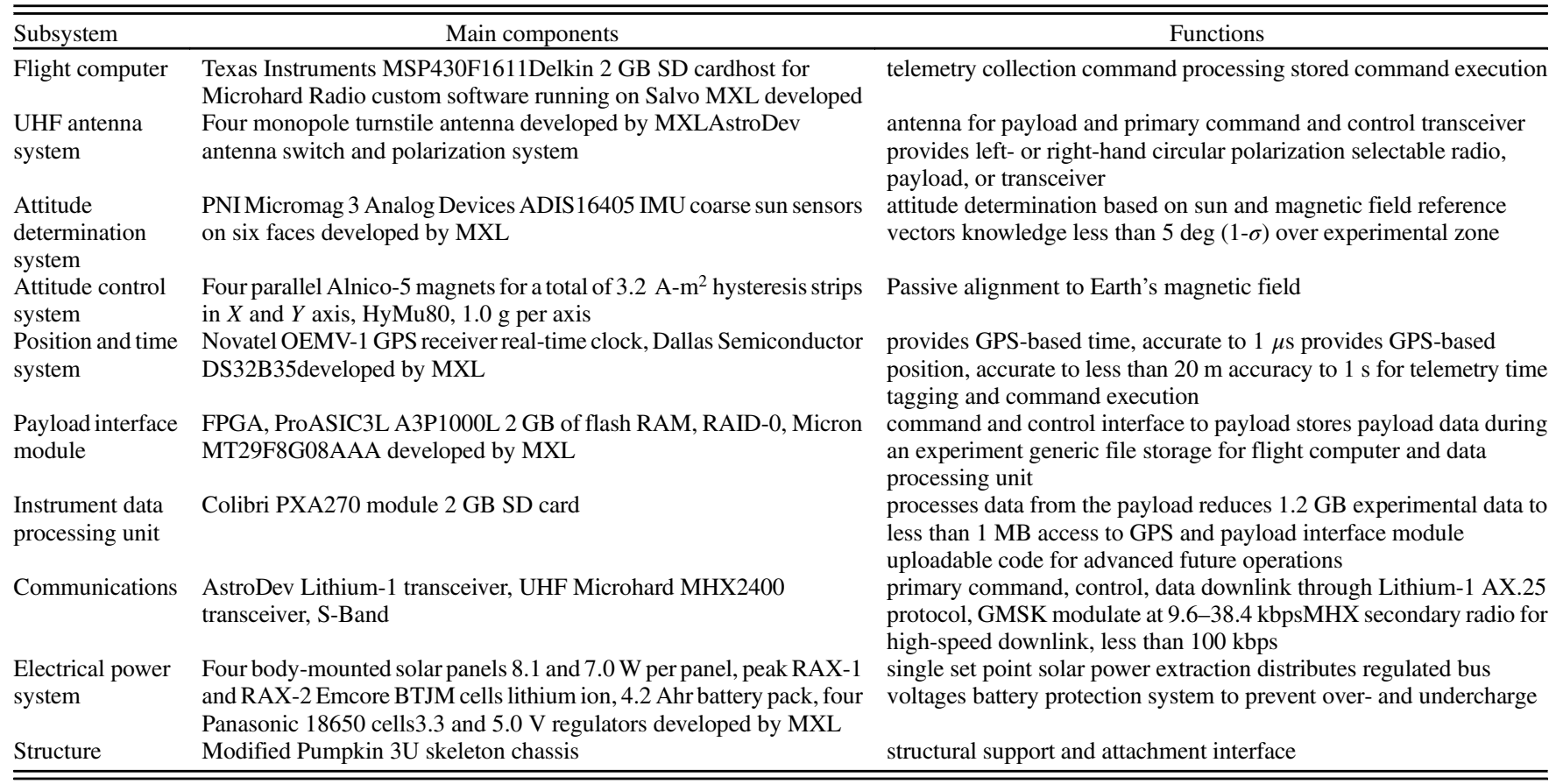




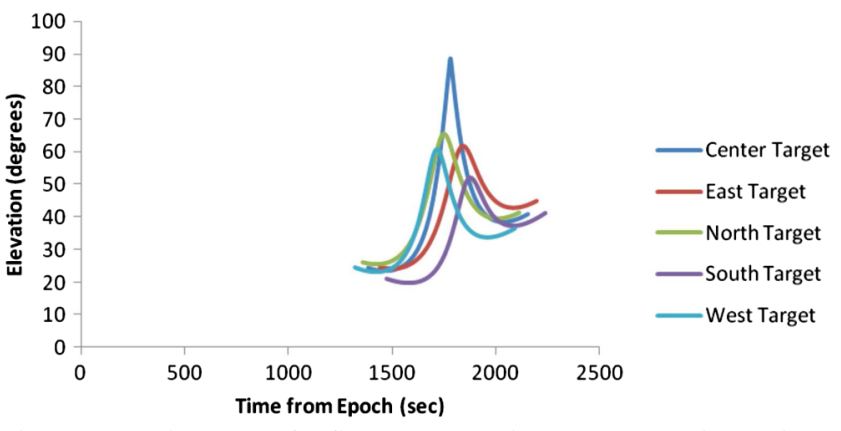

Fig. 6 Elevation angles for five representative radar scattering regions.

\section{Payload Data Collection and Processing}

Both the data rate and storage amount of experimental payload data were problematic for existing CubeSat communication and computing capabilities. The payload data samples are 16 bits wide (14 bits from the analog-to-digital converter and two extra status bits) output at $1 \mathrm{MHz}$ on each clock edge for an effective rate of $32 \mathrm{Mbps}$. Standard off-the-shelf CubeSat processors did not have sufficient bandwidth to process this payload output, thus necessitating a custom design. During each experiment, 1.2 GB of data samples are generated. Even though standard flash memory storage systems are capable of handling multiple experimental data sets, available CubeSat communication systems cannot downlink the data in sufficient time.

The first issue to be addressed was the collection of high-rate payload data. Initially, an architecture was considered that directly interfaced the payload output to the memory bus of a high-speed general-purpose processor by using appropriate input-output buffers. This would provide an integrated system with minimal additional circuitry, but also present several problems. Development required knowledge of complex processor bus timing and interfaces, and most students working on RAX do not have this capability. Also, interfacing to high-speed processors requires state-of-the-art testing equipment, and this was not accessible to the satellite design laboratory.

In light of these challenges, an architecture was developed that provided a level of indirection between the processor and the payload by means of a custom interface through a field programmable gate array (FPGA). The payload interface module was designed to receive data samples from the payload and store them in its dedicated flash-memory system. It time tags the data with GPS-based pulseper-second signals, and then provides a standard serial peripheral interface (SPI) to the high-speed instrument data processing unit. Thus, the interface was simplified to the processor by providing a buffer to the payload and a simple access method through the serial interface.

The mission cost of this interface architecture was additional electronics, adding $70 \mathrm{~g}$ to the satellite weight, well within the mass margins of the spacecraft. The interface module was also a lower power solution because it only consumed $0.18 \mathrm{~W}$, compared to the data processing unit's power consumption of $2.1 \mathrm{~W}$. As an added feature, the processing unit could be powered off during data collection. As a result of these changes, students experienced with gate array design were easily recruited due to the incorporation of training materials in the University of Michigan curriculum.

The second challenge posed by a large data set in each experiment was solved with the processing unit. It is a general-purpose processor, a Marvell PXA270, running a standard operating system, Linux. Radar data processing software was developed that measures the noise floor of the receiver, calculates the power spectral densities of received signals, and directly processes radar experimental data. The ground radar transmission times are logged with reference to GPS time. The time history of transmissions is then uploaded to the satellite, which allows the processing unit to filter the data for the range of interest. The received radar pulses are then extracted and analyzed on orbit, and data size is reduced by three to four orders of magnitude. Subsets of the raw data can also be downloaded.

\section{Subsystem Overview}

A brief overview of each of the subsystems is given next. Detailed subsystem designs and architectures will be provided in future publications.

\section{Flight Computer}

The RAX flight computer is a low-power embedded processor system based on the Texas Instruments MSP430 microprocessor, and was developed internally at MXL. It performs typical flight computer functions such as telemetry collection, ground command processing, experiment sequencing, scheduled command execution, data storage, and communication through the radio systems. An integrated Secure Digital (SD) card provides 2 GB of data storage. Custom SD-card drivers were written because off-the-shelf libraries were too large in terms of code space; the libraries would not fit on the MSP430 with the other flight-specific code.

An external watchdog timer system provides fault detection and recovery of flight computer transient failures. The watchdog monitors a heartbeat signal from the computer and reboots it if a failure is detected. It can also be commanded from the ground through the UHF radio to force power resets of the flight computer. The watchdog itself is periodically power cycled by a 555-timer circuit to clear any internal transient errors.

Flight software was custom written for RAX in C, and ran on the Salvo operating system. All available code space was used.

\section{UHF Antenna System}

A single UHF antenna system is shared between the payload receiver and the primary communication transceiver. The antennas, pictured in Fig. 4, are a turnstile array with four active elements. The elements are made from spring steel and deployed after launch by a flight computer command at a predetermined time. Their length, approximately $17.5 \mathrm{~cm}$, is tuned to match PFISR. The widths of the antennas were broadened from a narrow whip antenna in order to increase their bandwidth for accommodating the other radar frequencies. The antenna system has a $70 \mathrm{deg} 1 \mathrm{~dB}$ beamwidth because the science mission requires antenna gain knowledge within $1 \mathrm{~dB}$. The typical $3 \mathrm{~dB}$ point for beamwidth numbers is not used. The element angles and orientation are fixed after initial deployment from a stowed position.

The antennas are connected to a circular polarization network. The network is switchable on orbit between left- or right-hand polarization. There are two radio connection ports that can switch the antenna between the payload receiver and the communication transceiver. The radio not switched to the antenna is isolated and connected to a protective dummy load.

\section{Attitude Determination}

The satellite attitude is determined from onboard magnetometers, sun sensors, and a rate gyroscope [24]. Two three-axis magnetometers located internally provide a reference to Earth's magnetic field direction. Their dynamic range is sufficient to accommodate the DC bias of the control magnets. Attitude knowledge is then derived from expected magnetic field values based on RAX's location as identified by the onboard GPS receiver or through two-line elementbased position determination. Sun sensors on all six faces of the satellites provide an additional reference vector for attitude determination.

Magnetometer, sun sensor, and rate gyroscope data are processed on the ground using a multiplicative extended Kalman filter to estimate RAX attitude. This information is made available during science analysis to confirm proper antenna orientation with respect to target zones. When the sun illuminates RAX, the angular knowledge uncertainty is between 2 and $3 \mathrm{deg}$, which meets the $5 \mathrm{deg} 1 \sigma$ attitude determination requirement. In eclipse, the uncertainty increases to between 7 and $8 \mathrm{deg}$. Because of the location of the primary ground radar satiation, the majority of RAX scientific experiments will take place when RAX is in the sun, satisfying the attitude determination requirement. 


\section{Position and Time}

The position and time system provides spatial and temporal information for the satellite to improve resolution of the irregularities and their fine scale features. The primary position and time source is an onboard GPS receiver. The simulations show accuracy of position better than $20 \mathrm{~m}$ and timing to less than a microsecond. Detailed simulations of proper antenna placement in the context of the attitude control scheme have been performed to estimate expected on-orbit performance [25].

A secondary timing system, based on a real time clock, provides time synchronization when GPS is not available, such as during planned GPS poweroff for energy conservation. The clock provides millisecond accuracy and maintains time during satellite reboots and resets. A pulse-per-second signal, sourced by either the GPS or the clock, is distributed throughout the satellite to synchronize data collections. The payload interface module and attitude determination system then use the signal to time tag data collections.

A secondary ground-based position determination system uses publicly available two-line element sets published by the U. S. government. Two-line element-based position error is expected to be less than $1 \mathrm{~km}$ within several days after the element set epoch [26].

\section{Payload Interface Module}

The payload interface module receives high-speed digital data from the payload, stores it in flash-based memory, and provides access to the storage memory to both the instrument data processing unit and the flight computer. The interface module contains a gate array clocked at approximately $50 \mathrm{MHz}$. The frequency is slightly lower than $50 \mathrm{MHz}$ to prevent electromagnetic interference from $50 \mathrm{MHz}$ harmonics appearing in the science data. Data are stored to $4 \mathrm{~GB}$ of mirrored flash memory bank. It is packetized with checksums and time tags are inserted based on timing and pulse-persecond output from the position and time system.

The interface module is accessible by both the flight computer and the instrument data processing unit through dual SPI busses. It provides a shared storage system to enable large file transfer between the flight computer and the processing unit. The processing unit retrieves payload data from the interface module and copies processed data back to it. The flight computer retrieves these files and stores them on flight-computer-based storage for later downlink. In a similar manner, files are transferable from the flight computer to the processing unit in order to enable code updates on the processing unit.

\section{Instrument Data Processing Unit}

The instrument data processing unit processes radar data to reduce downlink requirements. Onboard processing sorts through time series receiver data stored on the payload interface module and extracts data with radar pulses of interest. The segmented data are then processed with radar analysis code to calculate signal-to-noise ratios and range delays. The processing unit then stores the data on the payload interface module for downlink by the flight computer to the science operations center. It also has direct interface to the GPS unit for enhanced configuration and monitoring of GPS performance.

\section{Communication}

Satellite commanding and data downlink is through UHF (436$438 \mathrm{MHz})$ and $\mathrm{S}$-Band $(2.4 \mathrm{GHz})$ radio systems. The primary radio system is a half duplex radio, the Lithium-1 from Astronautical Development (AstroDev). The Lithium-1 is configured to run at data rates of 9.6-40 kbps using Gaussian minimum shift keying (GMSK) modulation in the UHF amateur radio band. It is compatible with typical amateur-radio-class ground stations operated by the global amateur radio community. The radio shares the antenna system with the payload receiver through an antenna switch. The switch is controlled by the flight computer and also polarizes the antenna in either left- or right-hand circular polarization.

The secondary radio system operates at $2.4 \mathrm{GHz}$ using the Microhard MHX-2400, a spread spectrum point-to-point radio. The MHX-2400 has flight heritage on past CubeSat missions [27,28] but performance of the radio has been less than expected on these missions [29]. The baud rate was increased to the radio and updated configuration settings of the secondary radio in an attempt to improve on-orbit downlink rates. Ground testing showed rates of $50 \mathrm{kbps}$.

\section{Internal Electrical Interfaces}

The primary electrical interface on RAX is through the CubeSat kit header. Initially, several commercial components were baselined for RAX that used the header, and when these systems were removed from the design, the header remained. The header does not extend above the payload interface module because the payload requires a complete Faraday cage to reduce noise. Custom interfaces to the payload and boards above the payload were developed. Through the header, a low-speed $\mathrm{I}^{2} \mathrm{C}$ bus provides access to distributed telemetry sensors. An SPI bus (greater than $1 \mathrm{Mbps}$ ) interconnects the payload interface module, instrument data processing unit, flight computer, position and time system, and radio systems. The attitude determination system has a local SPI bus as well to communicate with distributed sensors on the solar panels.

\section{9. $\quad$ Structure}

The structure is a skeletonized $3 \mathrm{U}$ structure developed by Pumpkin. This folded sheet-metal aluminum exoskeleton provides integration surfaces and mounting points for external systems such as antennas, solar panels, and programming adapters for internal computers. The structure was modified to accommodate the GPS antenna, the UHF antennas, solar panel mounts, and payload supports.

\section{Electrical Power System}

The electrical power system provides regulated power at 3.3 and $5 \mathrm{~V}$. A higher voltage battery bus line is distributed to satellite subsystems as well. During eclipse, this bus voltage is at the battery voltage. When the solar panels are illuminated, the bus is regulated to approximately $8 \mathrm{~V}$. RAX uses a $7.4 \mathrm{~V}, 4.4$ amp-hour lithium ion battery pack. The battery is housed on a custom printed circuit board with an aluminum housing for mechanical constraint. Battery telemetry, including voltage, current, and temperature, is provided through the $\mathrm{I}^{2} \mathrm{C}$ interface.

The power system has power conversion regulators for the four body-mounted solar panels. The panels each contain Emcore triplejunction solar cells, with eight per panel on RAX-1 and seven per panel on RAX-2. The maximum power per panel is 8.1 and $7.0 \mathrm{~W}$ at $25^{\circ} \mathrm{C}$ for RAX-1 and RAX-2, respectively. A single set-point controller extracts energy from the solar panels. It determines the realized maximum current output of the panels. A peak power tracking system was not implemented due to constraints on development time. $\mathrm{I}^{2} \mathrm{C}$-accessible telemetry includes solar panel voltage and current, bus voltage and current, and temperatures.

\section{E. Ground Segment}

The mission ground segment consists of several globally distributed resources. The science operations center is located at SRI International in Menlo Park, California. The mission operations center is located at the University of Michigan in Ann Arbor, Michigan. Primary ground communication stations for commanding and downlinking data are located at both centers.

The science operations center coordinates science experiments for the RAX missions and monitors space weather for conditions that are expected to create irregularities. Data sources include satellite data, solar observations of coronal mass ejections, and ground-based magnetometers. The center schedules radar transmission time and provides satellite operators with start and stop times for RAX experiments. During an experiment, it collects data from the radar stations directly to monitor real-time conditions.

The mission operations center commands the satellite, downlinks data, and monitors spacecraft health. Custom software developed at MXL generates commands and decodes telemetry. Client software is installed at remote ground stations to collect and forward data to servers located at MXL. Operations focus on running experiments, 


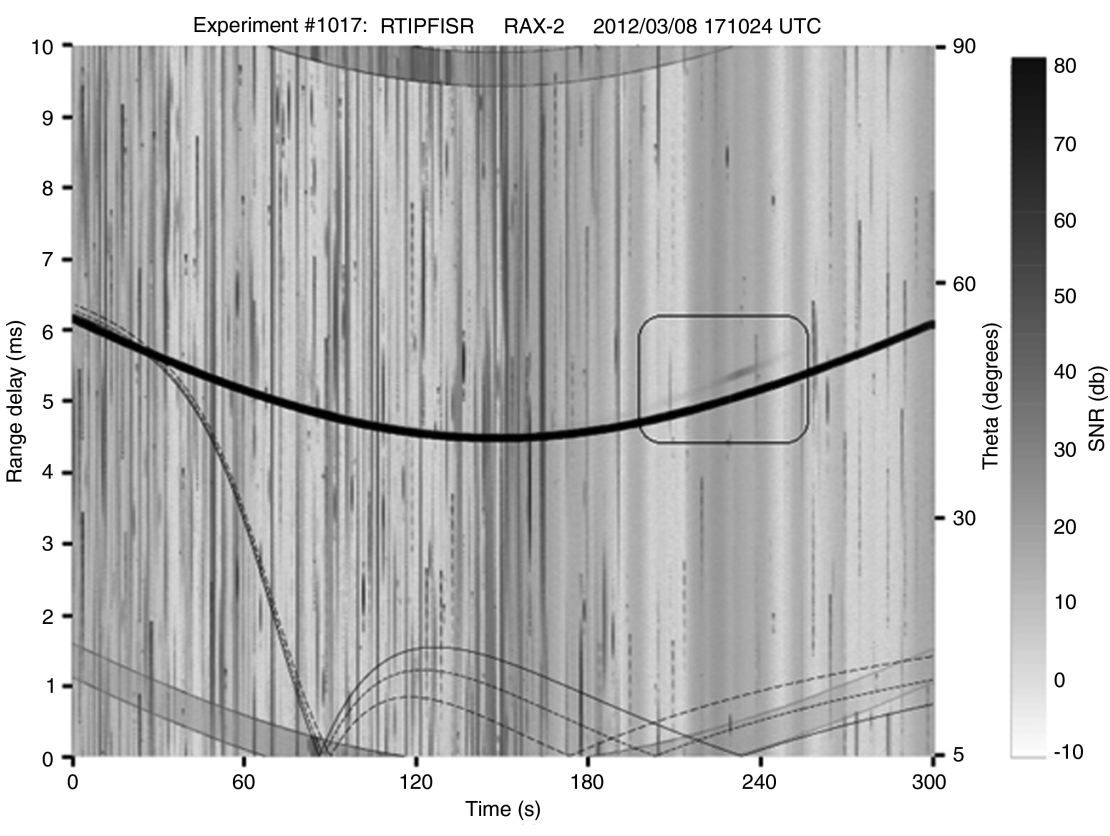

Fig. 7 Range-time-intensity plot from the RAX PFISR experiment conducted 8 March 2012.

downloading the data, and performing health assessment of the RAX satellites. Commanding ground station locations provide two to four commanding contacts per day. Telemetry is viewable to the general public online and payload data is shared with scientists.

\section{Flight Status}

The RAX mission currently consists of two satellites. RAX-1 was deployed on 19 November 2010 from a Minotaur-IV rocket launched from Kodiak, Alaska, as part of the STP-26 mission [30], into a circular orbit approximately $650 \mathrm{~km}$ in altitude and $72 \mathrm{deg}$ inclination. RAX-1 demonstrated on-orbit operation of the radar and satellite systems; however, its mission ended prematurely due to shadow-induced solar panel failure several months after launch. Initial analysis of the flight data has been published [31], and detailed analysis of the solar panel anomaly is currently under preparation for publication.
RAX-2 was launched on 28 October 2011 as part of the NASA CubeSat Launch Initiative with five other CubeSats. It was deployed into a $102 \mathrm{deg}$ inclination $400 \times 820 \mathrm{~km}$ altitude orbit by a Delta II rocket carrying NASA's NPP satellite. Vehicle checkout occurred during the first three weeks of operations and primary systems were found to be operational [32]. The first radar experiment was performed on 22 November 2011 with PFISR. In January 2012, the primary flash-based data storage system on the flight computer failed for an unknown reason. New code was uploaded to bypass the failure and nominal operations resumed a month later [32]. The S-Band radio was tested on 23 April 2012 at SRI International's $20 \mathrm{~m}$ dish in Stanford, California. Communication was successful; however, the effective throughput was comparable to the UHF radio system. Thus, due to the high cost of dish access to support the S-Band system and its low performance, all operations now use the UHF system. The $\mathrm{S}$-Band radio is periodically used as a heat source to keep battery

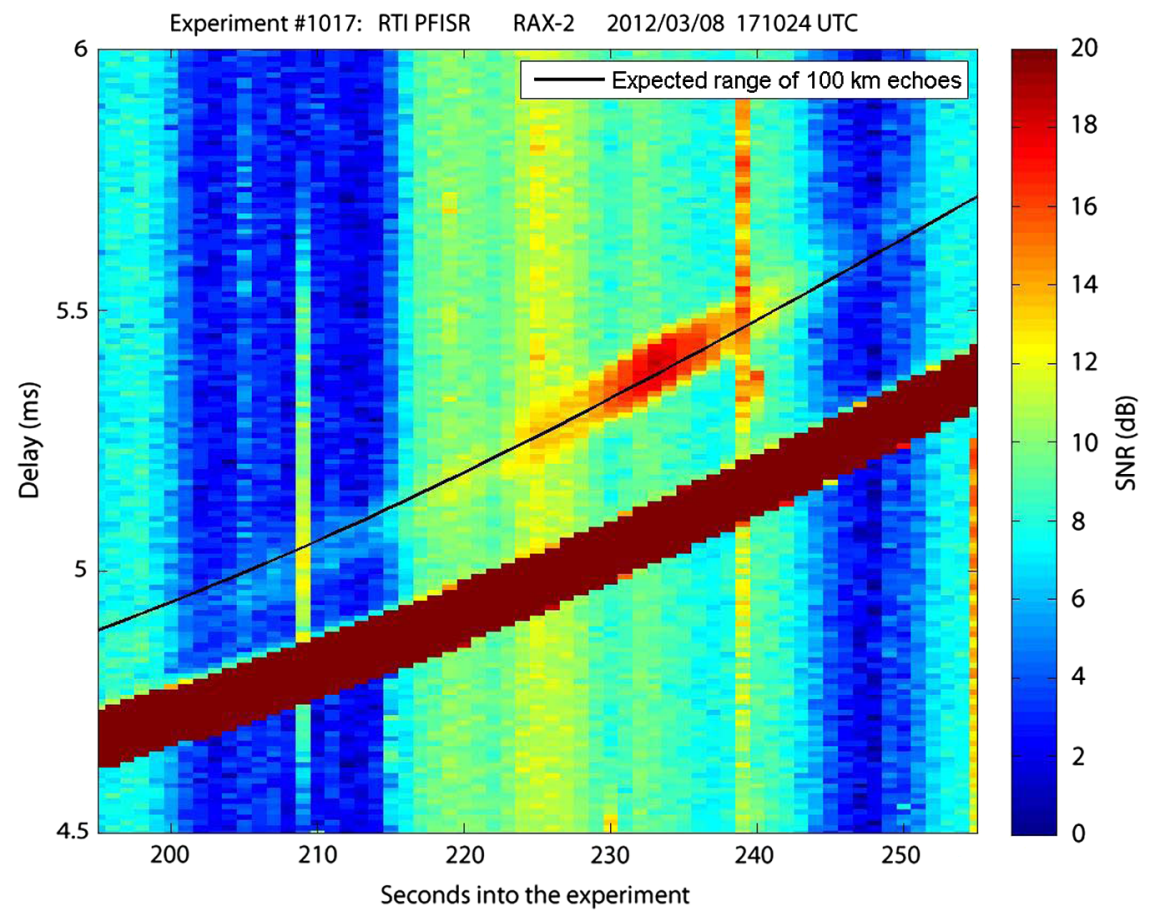

Fig. 8 Zoomed-in portion of Fig. 7 between 190 and $260 \mathrm{~s}$. 
temperatures at safe levels. The rotational kinetic energy of RAX-2 following deployment was dissipated by the passive magnetic system and reached steady state approximately three weeks after launch. Alignment to the geomagnetic field occurred approximately two months after launch. RAX-2 is scheduled to operate until end of life, which is expected in three to four years from when it was launched, when it will deorbit due to drag.

On 8 March 2012, RAX-2 recorded the first FAI-related echo [33]. The experiment was conducted with PFISR, and the resulting rangetime-intensity plot is shown in Fig. 7. The strip of saturated signal is a side lobe from the direct radar beam. Radar echo from irregularities is shown in the boxed region of the plot, just above the direct radar beam. The thin solid, dotted, and dashed lines show the aspect angles of scattering for the altitudes of 100,200 , and $300 \mathrm{~km}$, respectively. A zoomed-in portion of the data containing the radar echo is shown in Fig. 8. The black line is the expected range delay corresponding to irregülarities at an altitude of $100 \mathrm{~km}$. In this experiment, radar scatter was measured from irregularities at altitudes between 80 and $115 \mathrm{~km}$. Preliminary analysis performed in Bahcivan et al. [33] indicates the irregularities are located with an altitude resolution of $3 \mathrm{~km}$ and subdegree resolution in aspect angle. A thorough analysis is in progress for future publication.

As of 9 November 2012, after 378 days in orbit, RAX-2 has performed a total of 24 radar experiments with PFSIR, RISR, and MUIR. In three of these experiments, FAI were detected, which is consistent with other measured ionospheric properties taken during the experiments by ground systems. Also, during an experiment with MUIR, artificially generated FAI were measured due to ionospheric heating by HAARP. Even though originally 300 experiments were planned, far fewer have been performed due to downlink limitations. More raw and unprocessed data than expected are being downlinked, which has limited the number of experiments that can be performed and downlinked. Detailed science analysis is underway for these experiments and results will be published.

\section{Conclusions}

The Radio Aurora Explorer (RAX) mission was conceived to perform measurements of small-scale plasma irregularities, also known as field-aligned irregularities (FAI), in the ionosphere. The mission uses a novel experimental architecture where ground-based radars transmit high-powered pulses into the ionosphere that are then scattered into space and received by RAX satellites. The experimental configuration enables determination of the properties of the irregularities that have not been measurable before.

Two RAX satellites were developed by SRI International and the Michigan Exploration Laboratory at the University of Michigan. Both were launched and demonstrated radar measurements. Radar echo from the irregularities was first measured by the RAX mission on 8 March 2012, demonstrating the mission's technical capability to make these measurements despite the size and timeline constraints. This first measurement of echo has provided unprecedented characterization of FAI. The irregularities are located with an altitude resolution of $3 \mathrm{~km}$ and subdegree resolution in aspect angle, which is unprecedented in aural region measurements. The measurements from this experiment, as well as future RAX experiments, will enable improved characterization of meter-scale ionospheric irregularities.

\section{Acknowledgments}

This work was supported by National Science Foundation grant ATM-0838054 to SRI International and the University of Michigan. We thank Richard Behnke and Therese Moretto Jørgensen for their support in these space weather missions. They are enabling the frontier of space exploration. Thanks to Scott Schaire who helped navigate satellite integration. Thanks go to the U.S. Air Force Space Test Program and the NASA CubeSat Launch Initiative, which provided the rides to space. We thank all the Michigan students that worked on RAX through classes or volunteered. Matt Bennett, Andy Klesh, Ben Kempke, Alex Sloboda, and John Springmann, thank you for your leadership and heroic efforts. The administrative and technical staff in the Aerospace Department were instrumental in providing the support needed to keep the facilities, finances, and all other matters working well, thank you. Thanks to our engineering support manager, Allison Craddock, for keeping us on schedule, in the right location, caffeinated, and looking sharp. Kevin Brown, thanks for your help on radios and making sure we could talk to the satellites. We thank all the global HAMs who have helped receive packets from the RAX satellites, in particular Mike Rupprecht, Colin Hurst, Mark Hammond, and Alan Cresswell. We thank Mike Nicolls of SRI International for running Poker Flat Incoherent Scatter Radars at short notices and for providing and discussing the radar data. We thank John Buonocore, Karl Van Dyke, and Kyle Leveque who worked on the payload receiver development. Thanks to Michael Heywood for the help on the scatter angle plotting. Finally, we greatly appreciate Rick Doe's efforts to keep the RAX momentum moving forward throughout the mission and for spear heading effort to get RAX-2 proposed to the NASA CubeSat Launch Initiative.

\section{References}

[1] Moretto, T., "CubeSat Mission to Investigate Ionospheric Irregularities," Space Weather, Vol. 6, No. 11, Nov. 2008. doi:10.1029/2008SW000441

[2] Nason, I., Puig-Sair, J., and Twiggs, R. J., "Development of a Family of Picosatellite Deployers Based on the CubeSat Standard," Proceedings of IEEE Aerospace Conference, Vol. 1, Big Sky, Montana, 2002.

[3] Baker, D. N., and Worden, S. P., "The Large Benefits of Small-Satellite Missions," Eos, Transactions American Geophysical Union, Vol. 89, No. 33, 2008, pp. 301-302. doi:10.1029/2008EO330001

[4] Moretto, T., and Robinson, R. M., "Small Satellites for Space Weather Research," Space Weather, Vol. 6, No. 5, May 2008. doi:10.1029/2008SW000392

[5] Lanzerotti, L. J. (ed.), "Report of the Assessment Committee for the National Space Weather Program," Office of the Federal Coordinator for Meteorological Services and Supporting Research Rept. FCM-R242006, Silver Spring, MD, June 2006.

[6] Bahcivan, H., and Cutler, J. W., "Radio Aurora Explorer: Mission Science and Radar System," Radio Science, Vol. 47, No. 2, 2012, pp. 1-12. doi:10.1029/2011RS004817

[7] Farley, D. T., Jr., "A Plasma Instability Resulting in Field-Aligned Irregularities in the Ionosphere," Journal of Geophysical Research, Vol. 68, No. 22, 1963, pp. 6083-6097. doi:10.1029/JZ068i022p06083

[8] Buneman, O., "Excitation of Field Aligned Sound Waves by Electron Streams," Physical Review Letters, Vol. 10, No. 7, 1963, pp. 285-287. doi:10.1103/PhysRevLett.10.285

[9] Hoh, F., "Instability of Penning-Type Discharge," Physics of Fluids, Vol. 6, No. 8, 1963, 1184-1191. doi:10.1063/1.1706878

[10] Fejer, B. G., Farley, D. T., Balsley, B. B., and Woodman, R. F., "Vertical Structure of the VHF Backscattering Region in the Equatorial Electrojet and the Gradient Drift Instability," Journal of Geophysical Research, Vol. 80, No. 10, 1975, pp. 1313-1324. doi:10.1029/JA080i010p01313

[11] Bahcivan, H., Hysell, D. L., Larsen, M. F., and Pfaff, R. F., "The $30 \mathrm{MHz}$ Imaging Radar Observations of Auroral Irregularities During the Joule Campaign," Journal of Geophysical Research, Vol. 110, No. A5, May 2005, pp. 1-13. doi:10.1029/2004JA010975

[12] Kindel, J. M., and Kennel, C. F., "Topside Current Instabilities,” Journal of Geophysical Research, Vol. 76, No. 13, 1971, pp. 3055-3078. doi:10.1029/JA076i013p03055

[13] Cosgrove, R. B., Lu, G., Bahcivan, H., Matsuo, T., Heinselman, C. J., and McCready, M. A., "Comparison of AMIE-Modeled and Sondrestrom-Measured Joule Heating: A Study in Model Resolution and Electric Field-Conductivity Correlation," Journal of Geophysical Research, Vol. 114, No. A4, April 2009, pp. 1-15. doi:10.1029/2008JA013508

[14] Ott, E., and Farley, D. T., "Microinstabilities and the Production of Short-Wavelength Irregularities in the Auroral F Region," Journal of Geophysical Research, Vol. 80, No. 34, 1975, pp. 4599-4602. doi:10.1029/JA080i034p04599

[15] Rosenbluth, M. N., and Post, R. F., "High-Frequency Electrostatic Plasma Instability Inherent to "Loss-Cone" Particle Distributions," 
Physics of Fluids, Vol. 8, No. 3, 1965, pp. 547-550. doi: $10.1063 / 1.1761261$

[16] Post, R. F., and Rosenbluth, M. N., "Electrostatic Instabilities in Finite Mirror-Confined Plasmas," Physics of Fluids, Vol. 9, No. 4, 1966, pp. $730-749$. doi:10.1063/1.1761740

[17] Hultqvist, B., and Egeland, A., "Radio Aurora," Space Science Reviews, Vol. 3, No. 1, 1964, pp. 27-78. doi:10.1007/BF00226644

[18] Willis, N., Bistatic Radar, 2nd ed., SciTech Publishing, Raleigh, NC, 2005.

[19] Kelly, J. D., Heinselman, C. J., and Valentic, T., "A New Instrument for Ionospheric Research-AMISR," AGU Fall Meeting Abstracts, Vol. 1, 2006.

[20] Bahcivan, H., Kelley, M. C., and Cutler, J. W., "Radar and Rocket Comparison of UHF Radar Scattering from Auroral Electrojet Irregularities: Implications for a Nanosatellite Radar," Journal of Geophysical Research, Vol. 114, No. A6, June 2009, pp. 1-7. doi:10.1029/2009JA014132

[21] Matsuo, T., Richmond, A. D., and Nychka, D. W., "Modes of HighLatitude Electric Field Variability Derived from DE-2 Measurements: Empirical Orthogonal Function (EOF) Analysis," Geophysical Research Letters, Vol. 29, No. 7, 2002, pp. 11-1-11-4. doi:10.1029/2001GL014077

[22] Swartwout, M., "University-Class Satellites: From Marginal Utility to 'Disruptive' Research Platforms," 18th Annual AIAA/USU Conference on Small Satellites, Logan, UT, 2004.

[23] Bennett, M., Kloka, M., Ko, S., Prasad, R., and Underwood, N., "An Antenna for Space Weather Exploration Spacecraft," Electrical Engineering Computer Science 430 Final Report, Univ. of Michigan, Ann Arbor, MI, 2009.

[24] Springmann, J. C., Sloboda, A. J., Klesh, A. T., Bennett, M. W., and Cutler, J. W., "The Attitude Determination System of the RAX Satellite," Acta Astronautica, Vol. 75, June-July 2012, pp. 120-135. doi:10.1016/j.actaastro.2012.02.001
[25] Spangelo, S., Klesh, A., and Cutler, J., "Position and Time System for the RAX Small Satellite Mission," AIAA/AAS Astrodynamics Specialist Conference Proceedings, Paper 2010-7980, Toronto, 2010.

[26] Kelso, T., "Validation of SGP4 and IS-GPS-200d Against GPS Precision Ephemerides," Proceedings of the 17th AAS/AIAA Space Flight Mechanics Conference, Sedona, AZ, 2007.

[27] Yost, B., Engelbert, D., Hines, J., Agasid, E., Ricco, A., and Kitts, C., "The GENESAT-1 Test Demonstration Project: A Unique Use of Smallsats," Proceedings of 19th Annual AIAA/USU Conference on Small Satellites, Logan, UT, 2005.

[28] Kitts, C., Rozano, K., Rasay, R., Mas, I., and Acain, J., "Initial Flight Results from the PharmaSat Biological Microsatellite Mission," Proceedings of 23rd Annual AIAA/USU Conference on Small Satellites, Logan, UT, 2009.

[29] Mas, I., and Kitts, C., "A Flight-Proven 2.4 GHZ ISM-Band COTS Communications System for Small Satellites," Proceedings of 21rd Annual AIAA/USU Conference on Small Satellites, Logan, UT, 2007.

[30] Borowski, H., Reese, K., and Motola, M., "Responsive Access to Space: Space Test Program Mission S26," Proceedings of the IEEE Aerospace Conference, Big Sky, MT, 2010.

[31] Springmann, J., Cutler, J. W., and Bahcivan, H., "Initial Flight Results of the Radio Aurora Explorer," Proceedings of the 62nd International Astronautical Congress, Cape Town, South Africa, Oct. 2011.

[32] Springmann, J. C., Kempke, B. P., Cutler, J. W., and Bahcivan, H., "Development and Initial Operations of the RAX-2 CubeSat," The 4S Symposium, Portoroz, Slovenia, June 2012.

[33] Bahcivan, H., Cutler, J. W., Bennett, M., Kempke, B., Springmann, J. C., Buonocore, J., Nicolls, M., and Doe, R., "First Measurements of Radar Coherent Scatter by the Radio Aurora Explorer CubeSat," Geophysical Research Letters, Vol. 39, No. 14, July 2012, p. L14101. doi:10.1029/2012GL052249

R. Sedwick Associate Editor 